\title{
Caregiving burden and uplifts: A contradiction or a protective partnership for the quality of life of parents and their children with asthma?
}

\author{
Neuza Silva a - Carlos Carona a,c $\bullet$ Carla Crespo b - Maria Cristina Canavarro a \\ a Faculty of Psychology and Education Sciences, University of Coimbra, Coimbra, Portugal; \\ b Faculty of Psychology, University of Lisbon, Lisbon, Portugal; \\ c Coimbra Cerebral Palsy Association, Coimbra, Portugal
}

\begin{abstract}
Introduction. Parental caregiving in the context of pediatric chronic conditions is a multifaceted experience that encompasses negative (burdens) and positive dimensions (uplifts), which may support risk and protective processes that influence family adaptation. This study aimed to examine the caregiving experience of parents caring for a child with asthma and the moderating role of caregiving uplifts on the associations between caregiving burden and quality of life (QoL) of parents and their children. Method. Participants were 180 dyads of children/adolescents with asthma between 8 and 18 years of age and one of their parents. The parents reported on caregiving burden and uplifts and on their QoL, and the children/adolescents completed a self-report measure of generic QoL. Results. Results showed that although parents of children with intermittent asthma and parents of younger children presented higher levels of caregiving burden, non-controlled asthma was the only clinical variable representing a significant risk factor for decreased QoL in children. Significant negative and positive associations were found between burden dimensions and QoL and between caregiving uplifts and QoL, respectively, in parents and children. Additionally, caregiving uplifts moderated the negative link between relationship burden and parents' QoL. Conclusion. These results suggest that, far from being contradictory, caregiving uplifts may co-occur with high levels of burden and may constitute a protective factor against the deleterious effect of the caregiving burden on parents' QoL and a resource that directly contributes to children's QoL. This risk-resistance approach to family caregiving may contribute to operationalizing strength-based interventions in the context of pediatric asthma.
\end{abstract}

\section{Keywords}

Caregiving burden $\bullet$ Caregiving uplifts $\bullet$ Parent-child quality of life $\bullet$ Pediatric asthma $\bullet$ Risk and resistance factors

Silva, N., Carona, C., Crespo, C., \& Canavarro, M. C. (2015). Caregiving burden and uplifts: A contradiction or a protective partnership for the quality of life of parents and their children with asthma? Journal of Family Psychology, 29(2), I5I-I6I. doi:I0.1037/fam000007I 


\section{Introduction}

Pediatric chronic health conditions such as asthma are challenging contexts for parents, who, in addition to providing daily care to children as a normative task of parenting, must embrace the unexpected and stressful "caregiver career" (Raina et al., 2004). The burden resulting from caregiving demands, which include avoiding the environmental and emotional triggers of asthma exacerbation and monitoring symptoms and treatment (Fiese, Winter, Anbar, Howell, \& Poltrock, 2008), has been associated with quality of life (QoL) impairments for children with asthma and their parents (Crespo, Carona, Silva, Canavarro, \& Dattilio, 20II; Fiese, Wamboldt, \& Anbar, 2005). However, parental caregiving is a complex experience and the co-occurrence of uplifts (e.g., gratifications and positive emotions) arising from caring for a child with a chronic condition or disability has also been described (Green, 2007; Larson, 20I0). Although there is some evidence that caregiving uplifts may buffer the negative impact of the caregiving burden on QoL in parents of children with cerebral palsy (Carona, Pereira, Moreira, Silva, \& Canavarro, 20I3), few studies have examined this positive dimension of caregiving and its links to the adaptation outcomes in parents and their children with asthma. Understanding the role of caregiving uplifts may assist in the operationalization of a strength-based approach to pediatric asthma and may contribute to improving family adaptation in that context (Beresford, 1994; Judge, 1998).

Clinicians and researchers have long recognized that a pediatric chronic condition does not only affect the child; it also affects all family members and the relationships among them (Kazak, 1989). Although families facing such a chronic condition are at greater risk for psychosocial adaptation impairments, few children and parents present clinically significant psychological symptoms (Barlow \& Ellard, 2006). Moreover, studies comparing the QoL of children with asthma and their parents with healthy controls have yielded inconsistent findings (e.g., Moreira et al., 2013; Van Gent et al., 2007). To explain the wide variability in family adaptation trajectories, the disabilitystress-coping model highlights the importance of considering specific risk and resistance factors and the complex interactions between them (Wallander, Pitt, \& Mellins, 1990; Wallander, Varni, Babani, Banis, \& Wilcox, 1989). Within this model, disease parameters (e.g., severity), child functional dependence, and psychosocial stressors (e.g., daily hassles) have been proposed as important risk factors affecting children's and their parents' adaptation. In addition, the negative impact of diseasespecific risk factors has been hypothesized to be moderated by several resistance factors, namely intrapersonal factors (e.g., problem-solving ability), social-ecological factors (e.g., family environment) and stress processing mechanisms (e.g., cognitive appraisal).

Raina and colleagues (2004) also emphasized caregiving burden as an important risk factor for poor parental adaptation outcomes. The burden of caregiving has been broadly defined as the "caregiver's perceived responsibilities and associated limitations on self and family" (Canning, Harris, 
\& Kelleher, 1996, p. 737). Even if most research tends to use global measures resulting from the integration of different burden dimensions (Pinquart \& Sörensen, 2003), the caregiving burden should be further differentiated into relationship burden (i.e., disruptions in the relationship between the caregiver and the care receiver), objective burden (i.e., time constraints resulting from caregiving instrumental activities), and subjective burden (i.e., emotional stress and anxiety; Savundranayagam, Montgomery, \& Kosloski, 20II). This multi-dimensional approach is justified by the differential impact of burden dimensions on key outcomes. In fact, some authors found evidence for a direct association between the amount of assistance provided to a child during activities of daily living (i.e., objective burden) and the caregivers' psychological and physical health (Raina et al., 2005), while in other studies the subjective burden emerged as a foremost predictor of caregivers' distress (Canning et al., 1996) and QoL outcomes (Carona et al., 2013).

The associations between the additional practical demands and concerns arising from parenting a child with a chronic condition and the parents' impaired QoL are also well documented in empirical research conducted in the context of pediatric asthma (Crespo et al., 20I I; Fiese et al., 2005). However, little is known about the potential influences of parents' caregiving burden on their children's adaptation outcomes. The family is the primary context for child development, in general, and for the understanding and treatment of pediatric chronic conditions, in particular (Fiese \& Sameroff, 1989; Kazak, 1989). Moreover, a considerable amount of research has demonstrated that the children's ability to adapt to adversity is influenced by the quality of parental caregiving (Armstrong, Birnie-Lefcovitch, \& Ungar, 2005). Specifically, the parents' caregiving burden has been associated with impaired QoL for their children with asthma (Crespo et al., 20I I; Fiese et al., 2005), both directly and indirectly through mother-child interaction patterns (Fiese et al., 2008).

The disease parameters and child characteristics are also relevant risk factors, which can operate at different levels of the adaptation process. Disease severity has been associated with greater caregiving burden in parents caring for a child with a chronic condition (Canning et al., 1996). In the particular context of pediatric asthma, worse QoL has been found for parents and their children with greater asthma severity and poorly controlled symptoms (Dean et al., 2010; Everhart \& Fiese, 2009; Everhart, Fiese, \& Smyth, 2008). Although children's age has not been explicitly included in the disability-stress-coping model, there is some evidence of excessive time demands in providing care to children with chronic conditions or disabilities among mothers of younger children when compared with mothers of adolescents (Floyd \& Gallagher, 1997).

In addition to the deleterious effect of risk factors, research is now moving toward the study of how and when families can restore the balance between demands and capabilities to achieve the positive adaptation of vulnerable members and the family unit, in a context of significant adversity (McCubbin \& Patterson, 1983; Patterson, 2002; Walsh, 2002). Family psychological resources, which have been defined as "the family's characteristic mode of perceiving and interacting with the social 
world, including within and external to the family" (Wallander et al., 1989, p. 159), have assumed particular relevance in determining family adaptation in the context of pediatric chronic conditions. For example, research has consistently shown that a positive family environment is associated with better family adaptation outcomes in pediatric samples, in general (for a review, see Drotar, 1997), and in pediatric asthma, in particular (e.g., Silva, Crespo, \& Canavarro, 2014). In addition to operating as resources (i.e., they can positively influence adaptation outcomes regardless of the presence of a stressor), Wallander et al. (1989) have suggested that family variables may operate as protective factors (i.e., they can ameliorate negative outcomes or promote adaptive functioning under adverse conditions; Rose, Holmbeck, Coakley, \& Franks, 2004). The caregiving uplifts, as the parents' perceptions of gratifications and positive emotions arising from caregiving (Green, 2007; Larson, 2010), also fall within the definition of "family psychological resources". However, the role of caregiving uplifts as a resource contributing directly for the parents' and their children's QoL, or as a protective factor against the deleterious effects of caregiving burden, remains understudied for pediatric asthma.

Gratifications and positive emotions can emerge by attributing positive meanings to the caregiving experience, also known as the meaning-making process, which may occur at the global (e.g., reprioritization of family goals) or situational levels (e.g., benefit finding and personal growth; Park, 2010). Qualitative studies with families of children with developmental disabilities have described caregiving as an opportunity to develop new skills, widen interests, strengthen family relationships and expand personal and social networks, and as a source of personal growth (Grant, Ramcharan, McGrath, Nolan, \& Keady, 1998; Stainton \& Besser, 1998). A quantitative study with parents of children with cerebral palsy showed that the experience of caregiving uplifts directly contributed to better parental QoL and buffered the negative effect of the caregiving burden (Carona et al., 2013). Three adaptive functions of positive emotions occurring in stressful circumstances have been identified: broadening the "thought-action repertoire" and sustaining coping efforts, providing a "breather" or a distraction from daily hassles, and building new personal physical, intellectual, and social resources or restoring depleted ones (Folkman, 1997; Folkman \& Moskowitz, 2000; Fredrickson, 1998). Within family caregiving, parents' positive perceptions of their experience may buffer the negative impact of the child's health condition on family members and, ultimately, contribute to better QoL for parents and their children (Gupta \& Singhal, 2004).

The present study: Aims and hypotheses

Our study implemented a multi-dimensional approach to the caregiving experience, through the assessment of different components of caregiving burden and the examination of both burdens and uplifts as, respectively, risk and resource/protective factors for the adaptation of parents and their children with asthma. In the scope of the comprehensive concept of "adaptation", QoL has 
been defined as "a person's perception of his/her position in life within the context of the culture and value systems in which he/she lives and in relation to his/her goals, expectations, standards, and concerns" (The WHOQOL Group, 1994, p. 28). From this standpoint, we considered the parents' and their children's QoL as overall adaptation outcomes that reflect both the disease and the broader individual life experiences as influences on physical, psychological, and social well-being.

Based on a risk-resistance approach to family adaptation (Wallander et al., 1989, 1990), the objective of this study was twofold. First, we intended to describe the negative (burdens) and positive (uplifts) dimensions of family caregiving and the QoL of parents and their children in different clinical groups of asthma severity (intermittent vs. persistent) and control (controlled vs. partly controlled or uncontrolled), and at different developmental periods (childhood vs. adolescence). The second aim of our study was to examine the moderating role of caregiving uplifts on the associations between burden dimensions and the QoL of parents and their children. According to the conceptualization of disease parameters as risk factors for poor family adaptation outcomes, we expected higher levels of caregiving burden and worse QoL in the persistent and noncontrolled asthma groups $(\mathrm{HI})$. Considering that younger children require greater assistance in the activities of daily living and asthma management, we also predicted higher levels of caregiving burden and worse QoL among their parents compared with the parents of adolescents ( $\mathrm{H} 2)$. Given the scarce literature on the positive dimensions of caregiving, we made no predictions regarding the direction of group differences in caregiving uplifts. Moreover, we expected that caregiving burden would be negatively associated with parents' and their children's QoL $(H 3)$. Finally, based on the potential role of gratifications and positive emotions as protective factors against the adverse consequences of family stressors (Folkman, 1997; Gupta \& Singhal, 2004), we hypothesized that these associations would be moderated by caregiving uplifts (i.e., the negative associations between caregiving burden and QoL would be buffered by the parents' concomitant perceptions of caregiving uplifts; H4).

\section{Method}

\section{Participants}

The participants were 180 dyads of children and adolescents with asthma and one of their parents. The families were enrolled at the Pediatric and Immunoallergology outpatient services of three Portuguese public hospitals. Data collection took place between September, 2010 and February, 2012. For inclusion in the sample, the children/adolescents had to meet the following criteria: (a) between 8 and 18 years of age, (b) diagnosis of asthma established by a physician 
according to the International Classification of Diseases system (ICD-I0), (c) asthma diagnosis for at least one year, and (d) absence of comorbidities with other chronic health conditions or severe psychiatric disorders, except for comorbid allergic diseases (e.g., allergic rhinitis, atopic dermatitis/ eczema, and hypersensitivity to inhalant or food allergens). In addition, an inclusion criterion for parents was considered: being the parent self-identified as currently assuming the role of primary caregiver for the child in health-related issues. The participants' socio-demographic and clinical characteristics are presented in Table I.

Table I | Socio-demographic and clinical characteristics of the sample

\begin{tabular}{|c|c|c|c|}
\hline \multirow{2}{*}{\multicolumn{2}{|c|}{ Sorin }} & Parents $(n=180)$ & Children $(n=180)$ \\
\hline & eristics & & \\
\hline \multicolumn{2}{|c|}{ Age (in years), $M(S D)$} & 41.03 (5.77) & I I.98 (2.57) \\
\hline \multirow[t]{2}{*}{ Age group ${ }^{a}, n(\%)$} & Children $8-12$ years & - & $110(61.1 \%)$ \\
\hline & Adolescents $13-18$ years & - & 70 (38.9\%) \\
\hline \multirow[t]{2}{*}{ Gender, $n(\%)$} & Male & $28(15.6 \%)$ & I I 6 (64.4\%) \\
\hline & Female & 152 (84.4\%) & $64(35.6 \%)$ \\
\hline \multirow{4}{*}{$\begin{array}{l}\text { Socio-economic status }{ }^{b} \text {, } \\
n(\%)\end{array}$} & Low & \multicolumn{2}{|c|}{$106(58.9 \%)$} \\
\hline & Medium & \multicolumn{2}{|c|}{$58(32.2 \%)$} \\
\hline & High & \multicolumn{2}{|c|}{$14(7.8 \%)$} \\
\hline & Missing & \multicolumn{2}{|c|}{$2(1.1 \%)$} \\
\hline \multicolumn{4}{|l|}{ Clinical characteristics } \\
\hline \multirow[t]{4}{*}{ Asthma severity, $n(\%)$} & Intermittent & - & $95(52.8 \%)$ \\
\hline & Mild persistent & - & $52(28.9 \%)$ \\
\hline & Moderate persistent & - & $27(15.0 \%)$ \\
\hline & Severe persistent & - & $6(3.3 \%)$ \\
\hline \multirow[t]{3}{*}{ Asthma control, $n(\%)$} & Controlled & - & $120(66.7 \%)$ \\
\hline & Partly controlled & - & $5 \mathrm{I}(28.3 \%)$ \\
\hline & Uncontrolled & - & $9(5.0 \%)$ \\
\hline \multirow{3}{*}{$\begin{array}{l}\text { Comorbidities with other } \\
\text { allergic diseases, } n(\%)\end{array}$} & Allergic rhinitis & - & $13(7.2 \%)$ \\
\hline & Atopic dermatitis/eczema & - & 7 (3.9\%) \\
\hline & Unspecified allergies & - & $16(8.9 \%)$ \\
\hline \multicolumn{2}{|c|}{ Use of regular or emergency medication, $n(\%)$} & - & $176(97.8 \%)$ \\
\hline \multicolumn{2}{|c|}{ Length of time since asthma diagnosis (in years), $M(S D)$} & - & $7.36(3.95)$ \\
\hline
\end{tabular}

a The pediatric sample was divided into two age groups: children (8-12 years old) and adolescents (13-18 years old). For simplicity, we adopted the term children to refer to the pediatric sample, which includes both age groups. ${ }^{b}$ Socio-economic status was determined using a classification system for the Portuguese context based on the parents' job and educational level (Simões, 1994).

\section{Procedure}

The present study is part of a broader research project approved by the ethics committees and/or direction boards of three Portuguese public hospitals (Coimbra University Hospitals, Coimbra Pediatric Hospital, and Leiria Santo André Hospital). Using the non-probabilistic convenience sampling method, children who had routine medical appointments during the data collection period were screened by their physicians based on their medical files, and those who met the aforementioned criteria were invited to participate in the study. Detailed information about the 
study's aims and procedures was provided to all participants. Informed consent was obtained from parents and from adolescents older than 13 years, and informal assent was obtained from younger children. The parents and their children completed a set of self-report questionnaires in a room assigned to research purposes in the health institution they attended. A research assistant was available to provide support to participants with difficulties in reading or understanding the items' content.

\section{Measures}

\section{Caregiving burden and uplifts}

The Portuguese version of the Revised Burden Measure (Montgomery \& Kosloski, 2006; Portuguese version: ongoing psychometric studies) was used to assess the parents' experience of caregiving burden and uplifts. The parents were asked to indicate the extent to which various aspects of their lives had changed due to caregiving for their children with asthma ("As a result of assisting the care receiver, have the following aspects of your life changed?"), through 22 items clustered into three burden subscales and a distinct but complementary measure of caregiving uplifts. The Relationship burden subscale (five items) assesses the negative psychological state that directly derives from the caregiver's unique relationship with the care receiver, particularly as a result of demands for care and attention over and above the level that the caregiver perceives is warranted by the care receiver's condition (e.g., "Have your caregiving responsibilities increased attempts by your relative to manipulate you?"). The Objective burden subscale (six items) assesses the negative psychological state that results from the interference of caregiving activities on the caregiver's free time (e.g., "Have your caregiving responsibilities kept you from recreational activities?"). The Subjective burden subscale (five items) refers to a generalized form of negative affect resulting from caregiving, but not as a direct consequence of any specific event, task, or interaction (e.g., "Have your caregiving responsibilities created a feeling of hopelessness?"). The Uplifts measure comprises six items assessing the experience of gratifications and positive psychological states arising from caregiving, such as the direct enjoyment of caregiving tasks, an improved relationship with the child, and generalized positive affect (e.g., "Have your caregiving responsibilities given your life more meaning?"). All of the items were scored on a 5-point Likert scale ranging from I (not at all) to 5 ( $a$ great deal). Mean scores were calculated for each burden dimension and for the uplifts measure, with higher scores indicating the parents' perception of greater burden and more uplifts, respectively. Good internal consistency was obtained in our sample for all burden subscales, with Cronbach's alpha coefficients ranging from .84 (Subjective burden) to .88 (Objective burden), as well as for the Uplifts measure $(\alpha=.85)$. 
Parents' quality of life.

Parents' QoL was measured by the Portuguese version of the EUROHIS-QOL 8-item index (Pereira, Melo, Gameiro, \& Canavarro, 20II; Schmidt, Mühlan, \& Power, 2006). This self-report questionnaire is derived from the World Health Organization Quality of Life Assessment (WHOQOL-100 and WHOQOL-Bref instruments) and includes eight items representing the physical (e.g., "Do you have enough energy for everyday life?"), psychological (e.g., "How satisfied are you with yourself?"), social (e.g., "How satisfied are you with your personal relationships?"), and environmental (e.g., "How satisfied are you with the conditions of your living place?") domains of QoL. The eight items were scored on a 5-point Likert scale ranging, for instance, from not at all to completely. An overall QoL score was computed from the mean score of the items, with higher scores indicating better QoL. In the current sample, the questionnaire presented good reliability, with a Cronbach's alpha value of .83 .

\section{Children's quality of life.}

Children's generic QoL was measured by the Portuguese child-report version of the KIDSCREEN-10 Index (Matos, Gaspar, \& Simões, 2012; Ravens-Sieberer et al., 2010). This onedimensional questionnaire contains 10 items measuring physical well-being (e.g., "Have you felt fit and well?"), psychological well-being (e.g., "Have you felt sad?"), parent relations and autonomy (e.g., "Have your parent(s) treated you fairly?"), social support and peers (e.g., "Have you had fun with your friends?"), and school environment (e.g., "Have you got on well at school?"), and is answered on a 5-point Likert scale ranging from I (never/ not at all) to 5 (always/ extremely). A global score was calculated from the mean score of the items, with higher scores indicating better QoL. In our sample, the Cronbach's alpha coefficient was .78 , indicating good reliability.

\section{Socio-demographic and clinical data}

The asthma severity and control levels were classified by the child's physician according to the Global Initiative for Asthma (2008) guidelines. Asthma severity was categorized into four levels (intermittent, mild persistent, moderate persistent, and severe persistent), considering the level of symptoms, airflow limitation, and lung function variability. In addition to the clinical severity of the underlying disease, the physicians also classified the asthma control into three levels (controlled, partly controlled, and uncontrolled), in order to consider the responsiveness to treatment according to the asthma clinical manifestations, laboratory markers of inflammation, and pathophysiological features of the disease as recorded in the children's medical files.

Additional clinical data (e.g., use of medication, comorbidities with other health conditions, and length of time since asthma diagnosis) and socio-demographic information (e.g., parents' and their children's ages and genders, educational level and current job) were collected from the parents. 
Using a classification system specifically developed for the Portuguese context and based on the educational level and current job of the primary caregiver (Simões, 1994), the family socio-economic status (SES) was classified into low (e.g., unqualified employees in construction or manufacturing without completing the 9th grade of school education), medium (e.g., employees in bureaus or banks, nurses, social workers, or teachers with intermediate or university courses) and high (e.g., senior officials of government, army, commerce or industry, physicians, magistrates, or engineers with bachelor, master, doctorate, or other post-graduate degrees).

\section{Statistical analyses}

The statistical analyses were conducted with SPSS v.20.0 (SPSS Inc., Chicago, IL). Except for socio-demographic and clinical variables, missing data, which were random and less than $5 \%$ of the total values, were replaced with the individual mean score for each variable. Descriptive statistics were calculated for socio-demographic and clinical variables. Due to the heterogeneous distribution of family SES, asthma severity, and control levels observed in our sample, these variables were dichotomized and dummy-coded (family SES: 0 - low $[n=106]$, I - medium/high $[n=72]$; asthma severity: 0 - intermittent [ $n=95$ ], I - persistent [mild, moderate, or severe, $n=85$ ]; asthma control: 0 - controlled [ $n=120$ ], I - non-controlled [partly controlled or uncontrolled, $n=60$ ]). Differences in the mean scores of the study variables between age groups (children aged 8-12 vs. adolescents aged 13-18), asthma severity groups (intermittent vs. persistent), and asthma control groups (controlled vs. non-controlled) were tested with one-way multivariate analyses of covariance for caregiving burden dimensions and with one-way univariate analyses of covariance (ANCOVA) for caregiving uplifts, parents' QoL and children's QoL, while controlling for the remaining factors as well as family SES and length of time since asthma diagnosis. For caregiving burden, when multivariate effects were significant, univariate analyses were performed to examine which dimensions significantly differed between groups.

Prior to conducting the regression analyses, we computed Pearson's correlation coefficients between the main socio-demographic (parents' and children's ages and genders and family SES) and clinical variables (asthma severity, asthma control, length of time since asthma diagnosis, and comorbidities with other allergic diseases) and the other study variables. The socio-demographic and clinical variables that were significantly associated with one or both of the independent/moderator and dependent variables were included as covariates in the regression models (Mackinnon \& Luecken, 2008). Following the procedures recommended by Aiken and West (1991), hierarchical regression analyses were performed to examine the main and interaction effects of caregiving burden and uplifts on parents' and their children's QoL. To reduce multicollinearity with the interaction terms and to render the regression coefficients more meaningful and interpretable, the 
independent (burden dimensions) and moderator (uplifts) variables were mean centered before computing the interaction terms (Aiken \& West, 1991; Hayes, 2013). After the inclusion of covariates in the first step of the regression equation, the independent variables, the moderator, and the interaction terms were entered as predictors in subsequent steps. According to the guidelines for testing multiple moderating effects (Frazier, Tix, \& Barron, 2004), we introduced the interaction terms together in the same step of the regression equation. Using the Modgraph computational tool (Jose, 2013), the significant interaction effects were plotted from the estimates of the dependent variable at different values of the moderator, and the strength and significance of each regression line was analyzed with post-hoc simple slope computations.

\section{Results}

Descriptive statistics and analyses of covariance

Descriptive statistics for caregiving burden and uplifts and for parents' and children's QoL are presented in Table 2.

Table 2 | Descriptive statistics and univariate analyses of covariance by asthma severity, asthma control and children's age groups

\begin{tabular}{|c|c|c|c|c|}
\hline & \multicolumn{2}{|c|}{ Asthma severity } & & \\
\hline & Intermittent $(n=91)$ & Persistent $(n=84)$ & & \\
\hline & $M(S D)$ & $M(S D)$ & $F_{(1,169)}$ & $\eta_{p}^{2}$ \\
\hline Relationship burden & $\mathrm{I} .6 \mathrm{I}(0.77)$ & $1.40(0.57)$ & $4.67^{*}$ & .03 \\
\hline Objective burden & $1.77(0.76)$ & $1.80(0.77)$ & 0.00 & .00 \\
\hline Subjective burden & $2.05(0.87)$ & $1.99(0.83)$ & 0.08 & .00 \\
\hline Uplifts & $3.06(0.91)$ & $3.02(1.06)$ & 0.00 & .00 \\
\hline Parents' QoL & $3.75(0.54)$ & $3.78(0.5 \mathrm{I})$ & 0.83 & .01 \\
\hline Children's QoL & $4.34(0.48)$ & $4.29(0.56)$ & 0.04 & .01 \\
\hline & Asthn & control & & \\
\hline & Controlled $(n=116)$ & Non-controlled $(n=59)$ & & \\
\hline & $M(S D)$ & $M(S D)$ & $F_{(1,169)}$ & $\eta_{p}^{2}$ \\
\hline Relationship burden & $1.52(0.72)$ & $\mathrm{I} .49(0.63)$ & 0.40 & .00 \\
\hline Objective burden & $\mathrm{I} .77(0.73)$ & $\mathrm{I} .80(0.82)$ & 0.00 & .00 \\
\hline Subjective burden & $2.04(0.85)$ & $1.98(0.84)$ & 0.21 & .00 \\
\hline Uplifts & $3.08(0.98)$ & $2.97(0.99)$ & 0.58 & .00 \\
\hline Parents' QoL & $3.79(0.48)$ & $3.70(0.60)$ & 1.46 & .01 \\
\hline Children's QoL & $4.38(0.46)$ & $4.20(0.61)$ & $4.37^{*}$ & .03 \\
\hline
\end{tabular}




\begin{tabular}{|c|c|c|c|c|}
\hline & \multicolumn{2}{|c|}{ Age group } & & \\
\hline & Children $(n=107)$ & Adolescents $(n=68)$ & & \\
\hline & $M(S D)$ & $M(S D)$ & $F_{(1,169)}$ & $\eta_{p}^{2}$ \\
\hline Relationship burden & $1.57(0.68)$ & I.4I (0.69) & 3.32 & .02 \\
\hline Objective burden & $1.92(0.79)$ & $\mathrm{I} .57(0.66)$ & $9.91^{* *}$ & .06 \\
\hline Subjective burden & $2.11(0.85)$ & $1.89(0.82)$ & $4.92^{*}$ & .03 \\
\hline Uplifts & $3.15(0.93)$ & $2.88(1.05)$ & $4.34^{*}$ & .03 \\
\hline Parents' QoL & $3.72(0.50)$ & $3.83(0.56)$ & $5.51^{*}$ & .03 \\
\hline Children's QoL & $4.35(0.56)$ & $4.27(0.46)$ & 0.53 & .00 \\
\hline
\end{tabular}

$p \leq .05 ;{ }^{* *} p \leq .01$, two-tailed.

Significant multivariate effects of the asthma severity group, Wilks' $\lambda=.94 ; F_{(3,167)}=3.39, p=$ $.02 ; \eta_{p}^{2}=.06$, and the children's age group, Wilks' $\lambda=.94 ; F_{(3,167)}=3.48, p=.02 ; \eta_{p}^{2}=.06$, were found for caregiving burden. The univariate analyses (see Table 2) indicated higher levels of relationship burden for parents caring for a child with intermittent asthma compared with the persistent asthma group, and higher levels of objective and subjective burden for parents of younger children than for parents of adolescents. The ANCOVAs for caregiving uplifts and for parents' QoL also presented significant differences between the children's age groups, with parents of younger children reporting more uplifts but lower QoL than parents of adolescents. Regarding children's QoL, a significant difference between asthma control groups was found, with children with controlled asthma reporting better QoL than children with non-controlled asthma.

Main and interaction effects of caregiving burden and uplifts on parents' and their children's QoL

As presented in Table 3, preliminary correlation analyses indicated weak to moderate positive associations between burden dimensions and caregiving uplifts and negative associations between burden dimensions and parents' and their children's QoL. Conversely, caregiving uplifts were not significantly associated with parents' or children's QoL. Regarding socio-demographic and clinical characteristics, children's younger age, lower SES, and intermittent asthma were significantly associated with greater relationship burden; children's younger age was associated with greater objective burden; and low SES was associated with greater subjective burden. In addition, medium/high SES was correlated with parents' and their children's better QoL, and controlled asthma was positively associated with children's QoL (see Table 3). Accordingly, these variables were included as covariates in the regression analyses when appropriate. No significant associations were found for parents' age, parents' and children's gender, length of time since asthma diagnosis, and presence of comorbidities with other allergic diseases. 
Table 3 | Inter-correlations among study variables and socio-demographic and clinical characteristics

\begin{tabular}{|c|c|c|c|c|c|c|}
\hline \multirow[t]{2}{*}{ Psychosocial variables } & \multicolumn{3}{|c|}{ Burden dimensions } & \multirow{2}{*}{$\frac{\text { Uplifts }}{4}$} & \multicolumn{2}{|c|}{ QoL outcomes } \\
\hline & I & 2 & 3 & & 5 & 6 \\
\hline I. Relationship burden & - & & & & & \\
\hline 2. Objective burden & $.72^{* *}$ & - & & & & \\
\hline 3. Subjective burden & $.57^{* *}$ & $.66^{* *}$ & - & & & \\
\hline 4. Uplifts & $.16^{*}$ & $.30^{* *}$ & $.20^{* *}$ & - & & \\
\hline 5. Parents' QoL & $-.25^{* *}$ & $-.15^{*}$ & $-.34^{* *}$ & .13 & - & \\
\hline 6. Children's QoL & $-.30^{* *}$ & $-.20^{* *}$ & $-.24^{* *}$ & .12 & $.25^{* *}$ & - \\
\hline \multicolumn{7}{|c|}{ Socio-demographic and clinical variables } \\
\hline Children's age (in years) & $-.16^{*}$ & $-.27^{* *}$ & -.14 & -.14 & .12 & -.06 \\
\hline Children's gender ${ }^{a}$ & .01 & -.04 & .09 & .00 & -.03 & -.04 \\
\hline Parents' age (in years) & -.05 & -.10 & -.11 & -.10 & -.07 & -.03 \\
\hline Parents' gender ${ }^{a}$ & -.05 & .02 & .12 & -.04 & -.08 & .06 \\
\hline Family SES ${ }^{b}$ & $-.16^{*}$ & -.02 & $-.22^{* *}$ & -.07 & $.19^{*}$ & $.22^{* *}$ \\
\hline \multicolumn{7}{|c|}{ Length of time since asthma } \\
\hline diagnosis (in years) & -.02 & -.03 & $<.01$ & $<.01$ & -.11 & -.06 \\
\hline \multicolumn{7}{|l|}{ Comorbidities with other } \\
\hline allergic diseases $^{c}$ & .03 & .01 & $<.01$ & .04 & -.02 & .05 \\
\hline
\end{tabular}

${ }^{a}$ Children's and parents' gender: 0 - male; I - female. ${ }^{b}$ Family SES: 0 - low; I - medium/high. ${ }^{c}$ Comorbidities with other allergic diseases: 0 no; I - yes. " $p \leq .05 ;{ }^{* *} p \leq .01$, two-tailed.

Results from the regression analyses examining the main and interaction effects of the burden dimensions and caregiving uplifts on the parents' QoL are displayed in Table 4. Significant main effects of caregiving burden and uplifts were found, explaining $11 \%$ and $4 \%$ of the variance in parents' QoL, respectively. The main effects model showed that, while controlling for sociodemographic and clinical variables, the parents' experience of higher levels of subjective burden were significantly associated with lower QoL, and higher levels of caregiving uplifts significantly accounted for better QoL.

As depicted in Table 5, significant negative main effects of caregiving burden were also found on children's QoL, explaining $9 \%$ of its variance. Specifically, higher levels of relationship burden were associated with children's lower QoL. In addition, higher levels of caregiving uplifts were associated with children's better QoL, explaining $3 \%$ of its variance.

In addition to the main effects of caregiving burden and uplifts, the insertion of the interaction terms between these variables explained an additional variance of $5 \%$ in parents' QoL. Post hoc simple slope analyses (see Figure I) revealed that higher levels of relationship burden were associated with lower parental QoL when parents experienced low $(b=-.42$, SE $=.14 ; t=-2.97, p<$ $.0 \mathrm{I})$ or medium $(b=-.20, \mathrm{SE}=.08 ; t=-2.39, p=.02)$ levels of uplifts but not when they experienced high levels of uplifts $(b=-.02, \mathrm{SE}=.1 \mathrm{I} ; t=0.16, p=.87)$. 
Table 4 | Main and interaction effects of caregiving burden and uplifts on parents' QoL

\begin{tabular}{|c|c|c|c|c|c|c|c|c|}
\hline \multirow[b]{2}{*}{ Variables } & \multicolumn{2}{|c|}{$\begin{array}{l}\text { Step I: Covariates } \\
\Delta R^{2}=.05, \Delta F_{(3,174)}=3.26^{*}\end{array}$} & \multicolumn{2}{|c|}{$\begin{array}{l}\text { Step 2: Main effects of } \\
\text { burden dimensions } \\
\Delta R^{2}=.1 \mathrm{I}, \Delta F_{(3,171)}=7.35\end{array}$} & \multicolumn{2}{|c|}{$\begin{array}{l}\text { Step 3: Main effects of } \\
\text { caregiving uplifts } \\
\Delta R^{2}=.04, \Delta F_{(1,170)}=7.92\end{array}$} & \multicolumn{2}{|c|}{$\begin{array}{l}\text { Step 4: Interaction effects } \\
\Delta R^{2}=.05, \Delta F_{(3,167)}=3.44^{*}\end{array}$} \\
\hline & $b(S E)$ & $t$ & $b(S E)$ & $t$ & $b(S E)$ & $t$ & $b(S E)$ & $t$ \\
\hline Constant & $3.33(.20)$ & $16.99^{\text {*ata }}$ & $3.44(.19)$ & $17.88^{2029}$ & $3.40(.19)$ & $17.97^{* 2 * \pi}$ & $3.45(.19)$ & $18.43^{\text {kats }}$ \\
\hline Children's age & $.03(.02)$ & 1.77 & $.02(.02)$ & 1.55 & $.03(.02)$ & 1.74 & $.02(.02)$ & 1.40 \\
\hline Family SES a & $.21(.08)$ & $2.67^{* *}$ & $.10(.08)$ & 1.26 & $.12(.08)$ & 1.52 & $.10(.08)$ & 1.31 \\
\hline Asthma severity ${ }^{b}$ & $.03(.08)$ & 0.37 & $-.03(.08)$ & -0.35 & $-.02(.08)$ & -0.23 & $<-.01(.07)$ & -0.03 \\
\hline Relationship burden & & & $-.17(.08)$ & $-2.10^{*}$ & $-.15(.08)$ & -1.87 & $-.20(.08)$ & $-2.38^{*}$ \\
\hline Objective burden & & & $.19(.08)$ & $2.31^{*}$ & $.14(.08)$ & 1.67 & $.12(.08)$ & 1.47 \\
\hline Subjective burden & & & $-.21(.06)$ & $-3.60^{* * *}$ & $-.21(.06)$ & $-3.68^{* * * *}$ & $-.20(.06)$ & $-3.48^{* * *}$ \\
\hline Uplifts & & & & & $.11(.04)$ & $2.81^{* *}$ & . $16(.04)$ & $3.69^{* * * * *}$ \\
\hline Relationship burden $X$ Uplifts & & & & & & & $.22(.10)$ & $2.31^{*}$ \\
\hline Objective burden X Uplifts & & & & & & & $-.09(.08)$ & -1.11 \\
\hline Subjective burden $X$ Uplifts & & & & & & & $.07(.06)$ & 1.10 \\
\hline
\end{tabular}

${ }^{a}$ Family SES: 0 - low; I - medium/high. ${ }^{b}$ Asthma severity: 0 - intermittent; I - persistent. " $p \leq .05 ;{ }^{\prime \prime *} p \leq .01 ;{ }^{* * *} p \leq .001$, two-tailed. 
Table 5 | Main and interaction effects of caregiving burden and uplifts on children's QoL

\begin{tabular}{|c|c|c|c|c|c|c|c|c|}
\hline \multirow[b]{2}{*}{ Variables } & \multicolumn{2}{|c|}{$\begin{array}{l}\text { Step I: Covariates } \\
\Delta R^{2}=.07, \Delta F_{(4,173)}=3.4 I^{* *}\end{array}$} & \multicolumn{2}{|c|}{$\begin{array}{l}\text { Step 2: Main effects of } \\
\text { burden dimensions } \\
\Delta R^{2}=.09, \Delta F_{(3,170)}=6.40^{*}\end{array}$} & \multicolumn{2}{|c|}{$\begin{array}{l}\text { Step 3: Main effects of } \\
\text { caregiving uplifts } \\
\Delta R^{2}=.03, \Delta F_{(1,169)}=6.28^{*}\end{array}$} & \multicolumn{2}{|c|}{$\begin{array}{l}\text { Step 4: Interaction effects } \\
\Delta R^{2}=.01, \Delta F_{(3,166)}=0.8 \mathrm{I}\end{array}$} \\
\hline & $b(S E)$ & $t$ & $b(S E)$ & $t$ & $b(S E)$ & $t$ & $b(S E)$ & $t$ \\
\hline Constant & $4.37(.19)$ & $22.64^{2 * 3 * 4}$ & $4.56(.19)$ & $23.82^{\text {2**a }}$ & $4.52(.19)$ & $23.90^{\text {2*kF }}$ & $4.53(.19)$ & $23.67^{\text {kकk }}$ \\
\hline Children's age & $-.01(.02)$ & -0.56 & $-.02(.02)$ & -1.30 & $-.02(.02)$ & -1.16 & $-.02(.02)$ & -1.29 \\
\hline Family SES a & $.23(.08)$ & $2.91^{* * *}$ & $.15(.08)$ & 1.93 & $.17(.08)$ & $2.17^{*}$ & $.16(.08)$ & $2.01^{*}$ \\
\hline Asthma severity ${ }^{b}$ & $.03(.09)$ & 0.33 & $-.02(.08)$ & -0.27 & $-.02(.08)$ & -0.24 & $-.01(.08)$ & -0.16 \\
\hline Asthma control ${ }^{c}$ & $-.18(.09)$ & $-2.03^{*}$ & $-.18(.09)$ & $-2.07^{*}$ & $-.17(.09)$ & -1.96 & $-.17(.09)$ & $-2.01^{*}$ \\
\hline Relationship burden & & & $-.19(.08)$ & $-2.4 I^{*}$ & $-.18(.08)$ & $-2.22^{*}$ & $-.20(.08)$ & $-2.44^{*}$ \\
\hline Objective burden & & & $.03(.08)$ & 0.32 & $-.02(.08)$ & -0.23 & $-.04(.08)$ & -0.43 \\
\hline Subjective burden & & & $-.08(.06)$ & -1.34 & $-.08(.06)$ & -1.36 & $-.07(.06)$ & -1.10 \\
\hline Uplifts & & & & & $.10(.04)$ & $2.51^{*}$ & $.13(.04)$ & $2.85^{* *}$ \\
\hline Relationship burden $X$ Uplifts & & & & & & & $.1 \mathrm{I}(.10)$ & 1.12 \\
\hline Objective burden $X$ Uplifts & & & & & & & $<.01(.08)$ & 0.04 \\
\hline Subjective burden $X$ Uplifts & & & & & & & $-.01(.06)$ & -0.07 \\
\hline
\end{tabular}

${ }^{a}$ Family SES: 0 - low; I - medium/high. ${ }^{b}$ Asthma severity: 0 - intermittent; I - persistent. ' Asthma control: 0 - controlled; I - partly controlled/uncontrolled. ${ }^{*} p \leq .05 ;{ }^{* *} p \leq .01 ;{ }^{* * *} p \leq .00$ I, two-tailed. 


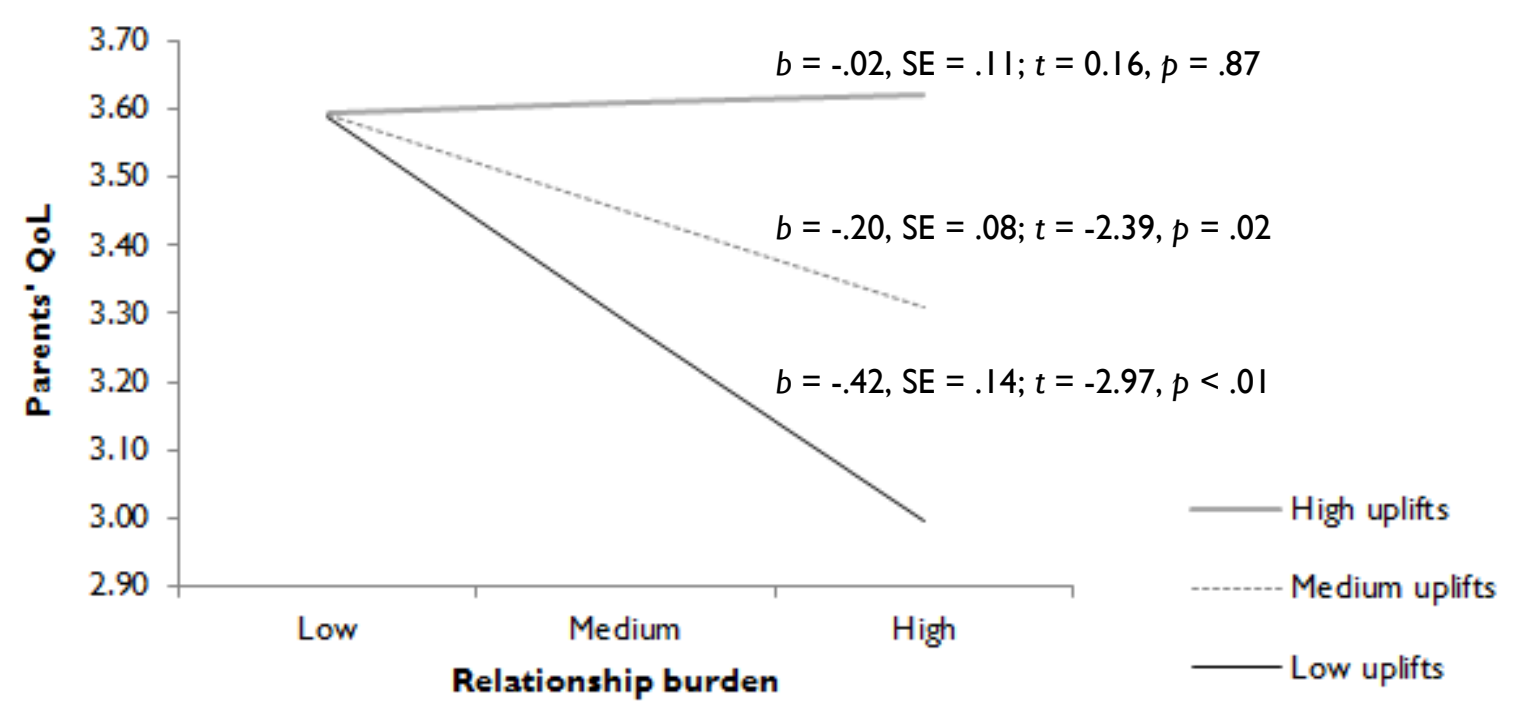

Figure I | The moderating effect of caregiving uplifts on the association between relationship burden and parents' QoL

No significant moderation effects of caregiving uplifts on the associations between objective or subjective burden and parents' QoL, or between any of the burden dimensions and children's QoL, were found.

\section{Discussion}

Grounded in the disability-stress-coping model (Wallander et al., 1989, 1990), the present study examined the caregiving experience and the QoL of parents and their children with different clinical characteristics of asthma and at different developmental stages, and the buffering role of caregiving uplifts on the negative associations between burden dimensions and QoL outcomes. Overall, our results showed that, although significant differences were found in caregiving dimensions according to asthma severity and children's age groups, these variables did not represent direct risk factors for parents' or their children's decreased QoL. In contrast, asthma control was not associated with the caregiving dimensions or with parents' adaptation, but it was a significant risk factor for children's QoL impairment. After controlling for clinical and socio-demographic variables, higher levels of caregiving burden were associated with worse QoL and higher levels of uplifts were associated with better QoL for both family members (main effects). In addition, caregiving uplifts moderated the negative association between relationship burden and parents' QoL (interaction effects). 
Our first set of results showed different effects of asthma clinical characteristics on the QoL outcomes of parents and children. Parents were more likely to perceive their children's demands for care and attention as over and above the needs warranted by the pediatric health condition when asthma was intermittent and did not inflict strict limitations on the children's functioning than when asthma was persistent. However, no significant effects of asthma severity or control were found on objective and subjective caregiving burdens or on parents' QoL. These results shall not exclude the possibility of indirect effects of asthma severity on parents' QoL, through its effect on caregiving burden dimensions. The hypothesis that intermittent asthma may result in increased relationship burden, which, in turn, negatively affects parents' QoL, needs further investigation. In contrast, and partially confirming our first hypothesis, children with controlled asthma reported better QoL than children with partly controlled or uncontrolled asthma. According to previous research reporting greater school/work absenteeism, lower productivity, and more impaired QoL for children with uncontrolled asthma and their caregivers when compared to the controlled asthma group (Dean et al., 2010; Schmier et al., 2007), significant differences in the caregiving burden dimensions and parents' QoL were also expected. The absence of significant differences between asthma control groups in our study may reflect the high portion of patients with partly controlled asthma that were included in the non-controlled asthma group, but it could also indicate that parents may be concerned with the broader aspects of their child's psychosocial functioning (Varni, Burwinkle, \& Lane, 2005) and constant involvement in asthma management routines (Fiese et al., 2008), regardless of asthma clinical characteristics.

Consistent with previous research on the time demands of caring for a child with a chronic condition or disability (Floyd \& Gallagher, 1997) and confirming our second hypothesis, parents of younger children presented higher levels of objective and subjective burden and lower QoL than parents of adolescents. These findings support the assumption that as the child ages and becomes more able to manage self-care routines, the responsibilities for asthma management are likely to be shared by family members, thereby alleviating caregiver burden (Kaugars, Klinnert, \& Bender, 2004; Kazak, 1989). Therefore, we advocate that conceptual models of family adaptation to pediatric chronic conditions should adopt a developmental perspective by including, among other aspects, children's younger age as a risk factor for parents' greater burden and impaired adaptation outcomes.

Interestingly, parents of younger children also presented more caregiving uplifts compared with parents of adolescents. In addition, higher levels of caregiving burden were associated with parents' perception of more uplifts. Taken together, these results suggest that parents are more likely to find benefits in the most stressful caregiving circumstances, which may reflect two different scenarios. On the one hand, and according to the differential susceptibility hypothesis (Belsky \& Pluess, 2009), some parents may be more vulnerable to environmental influences and, thus, more 
affected by both negative and positive features of their caregiving context. On the other hand, even if caring for a child with asthma is not considered a traumatic experience, it seems to include mechanisms similar to those underlying the genesis of post-traumatic growth (Tedeschi \& Calhoun, 2004), particularly if we take into account a recent study of breast cancer survivors showing that a negative life event may not necessarily be perceived as traumatic but may be stressful enough to shatter beliefs and expectations and to motivate a reappraisal of the stressful event and individual goals and capabilities (Silva, Moreira, \& Canavarro, 20II).

Regarding the second aim of our study, and confirming our third hypothesis, the results showed a negative contribution of caregiving burden dimensions, except for objective burden, to explain parents' and their children's QoL. These findings suggest that, more than objective risk factors, parents' subjective perceptions of stressor events and family relationships may determine coping processes and may ultimately influence their adaptation outcomes (Lazarus \& Folkman, 1984; Patterson, 2002). Regarding the positive effects of caregiving uplifts on QoL outcomes, different patterns have emerged for parents and children. For parents, caregiving uplifts were positively associated with their QoL and moderated the negative effect of relationship burden on QoL, whereas for children, only positive main effects of caregiving uplifts, but not moderating effects, were found. According to the conceptual distinction proposed by Rose et al. (2004), the perception of caregiving-related gratifications and positive emotions operated as a protective factor for the parents, that is, it contributed to buffering the negative effect of high levels of caregiving burden on QoL (moderation effect), and operated as a resource for the children, that is, contributed to better QoL, regardless of the presence of greater parental stress (main effect). A possible explanation of the moderation effects is the potential role of caregiving-related positive emotions in facilitating the development of positive meaning-based coping dispositions (e.g., positive reappraisal; Folkman, 1997; Fredrickson, 1998), which may serve as a buffer from emotional stress (Garro, 20I I).

In conclusion, our results strengthen the importance of addressing the caregiving burden as a multi-dimensional construct, including its relationship, objective, and subjective dimensions (Savundranayagam et al., 20II), because each dimension may be differentially related to both asthma clinical characteristics and parents' and their children's adaptation outcomes. In addition, to provide a comprehensive depiction of the parental caregiving experience, the positive dimension of caregiving and its effects on family adaptation should also be examined (Green, 2007; Larson, 20I0). Although it may seem contradictory at first, parents' perceptions of gratifications and positive emotions arising from caring for their children with asthma seems to occur in the most burdensome contexts (e.g., caring for a younger child). Finding positive meanings in the caregiving experience may constitute a protective factor against the deleterious effect of the caregiving burden on parents' QoL and an important resource that directly contributes to children's adaptation, thus benefiting all family members and fortifying the relational bonds among them (Walsh, 2002). 
Strengths and limitations

The main strength of our study was the innovative approach to the caregiving experience, including its negative and positive dimensions. To our knowledge, this is the first study to address the interaction effects of caregiving burden and uplifts on the QoL of parents and their children as a means of operationalizing a risk-resistance approach to family adaptation in the context of pediatric asthma. Our findings were strengthened by the inclusion of data from the child and his or her primary caregiver, which allows us to capture some of the complexity of family systems and to examine the inter-relationships among family members (Kazak, 1997). This methodological strategy was extended to the inclusion of data from individuals outside the family system (the child's physician, who reported on asthma severity and control), thus reducing the bias resulting from parent-reported asthma outcomes, which are likely to be influenced by parents' emotional status, perceptions, and expectations (Everhart \& Fiese, 2009; Kaugars at al., 2004; Kazak, 1997). In addition, the assessment of families living with pediatric asthma for at least one year sought to minimize the effect of acute stress responses caused by a recent diagnosis (Gupta \& Singhal, 2004) and to enable families to stabilize their functioning and meaning-making processes in this adverse context.

However, our findings should be interpreted with caution due to some limitations in the study's design and procedures. First, the study's cross-sectional design only allowed us to capture a snapshot of the ongoing dynamic process of family adaptation and precluded the establishment of causal links among study variables. Second, the non-probabilistic sample collection method and the heterogeneous distribution of socio-demographic and clinical characteristics of the sample may limit the generalizability of the results. The high percentage of children with intermittent and controlled asthma, although consistent with the distribution of asthma severity levels typically observed in the Portuguese pediatric population (Gaspar, Almeida, \& Nunes, 2006), required the dichotomization of asthma severity and control variables, which increased the intragroup variability and excluded the examination of interaction effects between age, severity, and control groups on the caregiving dimensions and QoL outcomes. Moreover, the great majority of caregivers was female and had low/medium SES, reflecting the users' profile in public pediatric healthcare services in Portugal. Even if the family SES was controlled in our analyses, the results should be interpreted within the Portuguese economic context, because the additional financial burdens may have influenced the caregiving experiences and, consequently, the parents' and their children's QoL outcomes. Third, only children with asthma and their primary caregivers were included, and the analyses were performed at the individual level (i.e., parents' and children's QoL were examined separately). Considering preliminary evidence showing that parents' and their children's QoL are interrelated 
(e.g., Everhart et al., 2008; Moreira et al., 2013; Silva et al., 20I4; Vila et al., 2003) and that the way in which families represent their experiences is based on transactional influences between family members over time (Fiese \& Sameroff, 1989), future research should adopt longitudinal designs, include other family members/units (e.g., couples and siblings) and rely on dyadic analyses of family adaptation processes. Finally, the parents' caregiving burden and uplifts contributed for explaining only a small amount of the variance in their own and their children's QoL. The child's characteristics (e.g., child behavior), caregiver's intrapersonal factors (e.g., self-esteem and sense of mastery over the caregiving situation), and coping resources (e.g., social support, family functioning, and stress processing mechanisms) have been acknowledged as highly relevant predictors of the caregiving experience and family adaptation outcomes (Fiese \& Sameroff, 1989; Raina et al., 2004) and, therefore, future research on this topic is warranted.

\section{Clinical implications}

The findings from this study have important implications for clinical interventions in pediatric asthma settings. In contrast to a healthcare approach focused only on the child and his or her health condition (Gupta \& Singhal, 2004), our results suggest that, in addition to symptom control, effective clinical interventions aimed at promoting family adaptation to pediatric asthma should be family centered and should encourage a positive outlook on caregiving demands and parent-child relationships. A risk-resistance approach to family adaptation (Wallander et al., 1989, 1990) offers a valuable conceptual map to identify and target key family processes that may reduce the risk of psychosocial problems, buffer stress, and even enable personal and relational growth in stressful contexts. In clinical practice, this framework is operationalized by strength-based interventions, particularly those focused on family meaning-making (e.g., positive reinterpretation of stressful events; appraisal of family capacities as sufficient to successfully manage distress) and coping strategies to withstand and rebound from adversity (Patterson, 2002; Walsh, 2002).

A multi-dimensional assessment and intervention targeting both negative (e.g., burdens) and positive (e.g., uplifts) dimensions of parental caregiving may contribute to operationalizing this strength-based approach to family adaptation. Therefore, it is important to avoid pathologizing parents' experience of gratifications and positive emotions when caring for a child with a health condition (e.g., considering them a form of denial of their child's condition) and to understand their adaptive function (Larson, 2010). Encouraging and supporting parents' perceptions of gratifications and positive emotions arising from caregiving may sustain adaptive coping processes (Folkman, 1997) and contribute to adaptation for parents and their children. Identifying thoughts and beliefs that interfere with positive experiences, facilitating coping strategies based on positive reappraisal, acceptance, and problem-solving, planning positive meaningful events, and infusing ordinary events 
with positive meaning (Folkman, 1997; Folkman \& Moskowitz, 2000) are specific intervention strategies that may help parents meet the challenge of "embracing the paradox" of their caregiving experience and perceive gratifications and positive emotions while they are also regulating distress (Carona et al., 2013; Larson, 1998).

\section{Acknowledgments}

This study is part of the "Growing up with asthma: Individual and family adaptation to pediatric asthma" research project, which is integrated in the Relationships, Development \& Health Research Group of the R\&D Unit Cognitive-Behavioral Center for Research and Intervention (Grant PEst-OE/PSI/UI0730/2014). Neuza Silva was supported by a PhD Scholarship from the Portuguese Foundation for Science and Technology (Grant SFRH/BD/69885/20I0). 


\section{References}

Aiken, L., \& West, S. (199I). Multiple regression: Testing and interpreting interactions. Thousand Oaks, CA: Sage.

Armstrong, M. I., Birnie-Lefcovitch, S., \& Ungar, M. T. (2005). Pathways between social support, family well-being, quality of parenting, and child resilience: What we know. Journal of Child and Family Studies, 14, 269-28I. doi:10.1007/s 10826-005-5054-4

Barlow, J. H., \& Ellard, D. R. (2006). The psychosocial well-being of children with chronic disease, their parents and siblings: An overview of the research evidence base. Child: Care, Health \& Development, 32, 19-31. doi:10.1 I I I/j. I365-2214.2006.0059I.x

Belsky, J., \& Pluess, M. (2009). Beyond diathesis stress: Differential susceptibility to environmental influences. Psychological Bulletin, 135, 885-908. doi:10.1037/a0017376

Beresford, B. A. (1994). Resources and strategies: How parents cope with the care of a disabled child. Child Psychology \& Psychiatry \& Allied Disciplines, 35, 171-209. doi:10.1III/j.14697610.1994.tb0II36.x

Canning, R. D., Harris, E. S., \& Kelleher, K. J. (1996). Factors predicting distress among caregivers to children with chronic medical conditions. Journal of Pediatric Psychology, 21, 735-749. doi:I0.1093/jpepsy/2I.5.735

Carona, C., Pereira, M., Moreira, H., Silva, N., \& Canavarro, M. C. (2013). The disability paradox revisited: Quality of life and family caregiving in pediatric cerebral palsy. Journal of Child and Family Studies, 22, 97I-986. doi:10.1007/s 10826-012-9659-0

Crespo, C., Carona, C., Silva, N., Canavarro, M. C., \& Dattilio, F. (20II). Understanding the quality of life for parents and their children who have asthma: Family resources and challenges. Contemporary Family Therapy, 33, 179-196. doi:10.1007/s 1059I-01 I-9155-5

Dean, B. B., Calimlim, B. C., Sacco, P., Aguilar, D., Maykut, R., \& Tinkelman, D. (2010). Uncontrolled asthma: Assessing quality of life and productivity of children and their caregivers using a cross-sectional Internet-based survey. Health and Quality of Life Outcomes, 8, 96. doi: 10.1 186/1477-7525-8-96

Drotar, D. (1997). Relating parent and family functioning to the psychological adjustment of children with chronic health conditions: What have we learned? What do we need to know? Journal of Pediatric Psychology, 22, 149-165. doi:10.1093/jpepsy/22.2.149

Everhart, R. S., \& Fiese, B. H. (2009). Asthma severity and child quality of life in pediatric asthma: A systematic review. Patient Education and Counseling, 75, 162-168. doi:10.1016/j.pec.2008.10.001

Everhart, R. S., Fiese, B. H., \& Smyth, J. M. (2008). A cumulative risk model predicting caregiver quality of life in pediatric asthma. Journal of Pediatric Psychology, 33, 809-818. 
doi:10.1093/jpepsy/jsn028

Fiese, B. H., \& Sameroff, A. J. (1989). Family context in pediatric psychology: A transactional perspective. Journal of Pediatric Psychology, 14, 293-3/4. doi:10.1093/jpepsy/14.2.293

Fiese, B. H., Wamboldt, F. S., \& Anbar, R. D. (2005). Family asthma management routines: Connections to medical adherence and quality of life. The Journal of Pediatrics, I46, I7I-I76. doi:10.1016/j.jpeds.2004.08.083

Fiese, B. H., Winter, M., Anbar, R., Howell, K., \& Poltrock, S. (2008). Family climate of routine asthma care: Associating perceived burden and mother-child interaction patterns to child well-being. Family Process, 47, 63-79. doi:10.1 I I I/j.1545-5300.2008.00239.x

Floyd, F., \& Gallagher, E. (1997). Parental stress, care demands, and use of support services for school-age children with disabilities and behavior problems. Family Relations, 46, 359-37I. doi: $10.2307 / 585096$

Folkman, S. (1997). Positive psychological states and coping with severe stress. Social Science \& Medicine, 45, 1207-1221. doi:10.1016/S0277-9536(97)00040-3

Folkman, S., \& Moskowitz, J. T. (2000). Stress, positive emotion, and coping. Current Directions in Psychological Science, 9, II5-II8. doi:10.1। II/1467-8721.00073

Frazier, P. A., Tix, A. P., \& Barron, K. E. (2004). Testing moderator and mediator effects in counseling psychology research. Journal of Counseling Psychology, 5I, II5-134. doi:10.1037/0022-0167.51.1.115

Fredrickson, B. L. (1998). What good are positive emotions? Review of General Psychology, 2, 300-319. doi: 10.1037/1089-2680.2.3.300

Garro, A. (20II). Coping patterns in Latino families of children with asthma. Journal of Pediatric Health Care, 25, 347-354. doi:10.1016/j.pedhc.2010.04.005

Gaspar, A., Almeida, M., \& Nunes, C. (2006). Epidemiologia da asma grave [Epidemiology of severe asthma]. Revista Portuguesa de Imunoalergologia, I4(S2), 27-4I.

Global Initiative for Asthma. (2008). Global strategy for asthma management and prevention - Updated 2008. Retrieved from http://www.ginasthma.org

Grant, G., Ramcharan, P., McGrath, M., Nolan, M., \& Keady, J. (1998). Rewards and gratifications among family caregivers: Towards a refined model of caring and coping. Journal of Intellectual Disability Research, 42, 58-7I. doi:10.1046/j.I365-2788.1998.00079.x

Green, S. E. (2007). "We're tired, not sad": Benefits and burdens of mothering a child with a disability. Social Science \& Medicine, 64, I50-163. doi:10.1016/j.socscimed.2006.08.025

Gupta, A., \& Singhal, N. (2004). Positive perceptions in parents of children with disabilities. Asia Pacific Disability Rehabilitation Journal, 15(I), 22-35.

Hayes, A. F. (2013). An introduction to mediation, moderation, and conditional process analysis: A regression-based approach. New York, NY: Guilford. 
Jose, P. (2013). ModGraph-I: A programme to compute cell means for the graphical display of moderational analyses: The internet version. Wellington, New Zealand: Victoria University of Wellington. Retrieved from http://pavlov.psyc.vuw.ac.nz/paul-jose/modgraph/

Judge, S. L. (1998). Parental coping strategies and strengths in families of young children with disabilities. Family Relations, 47, 263-268. doi:10.2307/584976

Kaugars, A. S., Klinnert, M. D., \& Bender, B. G. (2004). Family influences on pediatric asthma. Journal of Pediatric Psychology, 29, 475-49I. doi:10.1093/jpepsy/jsh05I

Kazak, A. E. (1989). Families of chronically ill children: A systems and social-ecological model of adaptation and challenge. Journal of Consulting and Clinical Psychology, 57, 25-30. doi:10.1037/0022-006X.57.1.25

Kazak, A. E. (1997). A contextual family/systems approach to pediatric psychology: Introduction to the special issue. Journal of Pediatric Psychology, 22, I4I-I48. doi:I0.1093/jpepsy/22.2.14I

Larson, E. (1998). Reframing the meaning of disability to families: The embrace of paradox. Social Science \& Medicine, 47, 865-875. doi:10.1016/S0277-9536(98)00II3-0

Larson, E. (2010). Psychological well-being and meaning-making when caregiving for children with disabilities: Growth through difficult times or sinking inward. OTJR: Occupation, Participation and Health, 30, 78-86. doi:10.3928/I5394492-20100325-03

Lazarus, R., \& Folkman, S. (1984). Stress, appraisal, and coping. New York, NY: Springer.

MacKinnon, D. P., \& Luecken, L. J. (2008). How and for whom? Mediation and moderation in health psychology. Health Psychology, 27, 99-100. doi:10.1037/0278-6133.27.2(Suppl.).S99

Matos, M. G., Gaspar, T., \& Simões, C. (2012). Health-related quality of life in Portuguese children and adolescents. Psicologia: Reflexão e Crítica, 25, 230-237. doi:10.1590/S010279722012000200004

McCubbin, H. I., \& Patterson, J. M. (1983). The family stress process: The double ABCX model of adjustment and adaptation. Marriage \& Family Review, 6, 7-37. doi:I0.1300/J002v06n0I_02

Montgomery, R. J. V., \& Kosloski, K. (2006). The league of experienced family caregivers: Measure development. Milwaukee, WI: University of Wisconsin-Milwaukee.

Moreira, H., Carona, C., Silva, N., Frontini, R., Bullinger, M., \& Canavarro, M. C. (2013). Psychological and quality of life outcomes in pediatric populations: A parent-child perspective. Journal of Pediatrics, I63, 147I-I478. doi:10.1016/j.jpeds.2013.06.028

Park, C. L. (2010). Making sense of the meaning literature: An integrative review of meaning making and its effects on adjustment to stressful life events. Psychological Bulletin, 136, 257-30I. doi:10.1037/a0018301

Patterson, J. M. (2002).Understanding family resilience. Journal of Clinical Psychology, 58, 233-246. doi: $10.1002 /$ jclp. 10019

Pereira, M., Melo, C., Gameiro, S., \& Canavarro, M. C. (20II). Estudos psicométricos da versão em 
Português Europeu do índice de qualidade de vida EUROHIS-QOL-8 [Psychometric studies of the European Portuguese version of the EUROHIS-QOL-8 quality of life index]. Laboratório de Psicologia, 9(2), 109-123.

Pinquart, M., \& Sörensen, S. (2003). Associations of stressors and uplifts of caregiving with caregiver burden and depressive mood: A meta-analysis. The Journals of Gerontology: Series B: Psychological Sciences and Social Sciences, 58, II 2-128. doi:I0.1093/geronb/58.2.PI I2

Raina, P., O’Donnell, M., Rosenbaum, P., Brehaut, J., Walter, S. D., Russell, D., ... Wood, E. (2005). The health and well-being of caregivers of children with cerebral palsy. Pediatrics, II5, 626636. doi:10.1542/peds.2004-1689

Raina, P., O’Donnell, M., Schwellnus, H., Rosenbaum, P., King, G., Brehaut, J., ... Wood, E. (2004). Caregiving process and caregiver burden: Conceptual models to guide research and practice. BioMed Central Pediatrics, 4, I. doi:I0.I I86/I47|-243 I-4-I

Ravens-Sieberer, U., Erhart, M., Rajmil, L., Herdman, M., Auquier, P., Bruil, J., ... The European KIDSCREEN Group. (2010). Reliability, construct and criterion validity of the KIDSCREENI0 score: A short measure for children and adolescents' well-being and health-related quality of life. Quality of Life Research, 19, I487-I500. doi:10.1007/s I I 36-010-9706-5

Rose, B. M., Holmbeck, G. N., Coakley, R. M., \& Franks, E. A. (2004). Mediator and moderator effects in developmental and behavioral pediatric research. Journal of Developmental and Behavioral Pediatrics, 25, 58-67. doi:10.1097/00004703-200402000-00013

Savundranayagam, M. Y., Montgomery, R. J. V., \& Kosloski, K. (20II). A dimensional analysis of caregiver burden among spouses and adult children. The Gerontologist, 5I, 32I-33I. doi:10.1093/geront/gnq102

Schmidt, S., Mühlan, H., \& Power, M. (2006). The EUROHIS-QOL 8-item index: Psychometric results of a cross-cultural field study. European Journal of Public Health, 16, 420-428. doi:I0.1093/eurpub/ckil55

Schmier, J. K., Manjunath, R., Halpern, M. T., Jones, M. L., Thompson, K., \& Diette, G. B. (2007). The impact of inadequately controlled asthma in urban children on quality of life and productivity. Annals of Allergy, Asthma \& Immunology, 98, 245-25I. doi:I0.1016/SI08I-I206(10)607I 3-2

Silva, N., Crespo, C., \& Canavarro, M. C. (20I4). Transactional paths between children and parents in pediatric asthma: Associations between family relationships and adaptation. Journal of Child and Family Studies, 23, 360-372. doi: I0.1007/s 10826-0I3-9734-I

Silva, S., Moreira, H., \& Canavarro, M. C. (20II). Growing after breast cancer: A controlled comparison study with healthy women. Journal of Loss and Trauma, 16, 323-340. doi:I0.1080/I5325024.20II.572039

Simões, M. R. (1994). Investigações no âmbito da aferição nacional do teste das Matrizes Progressivas de Raven [Raven's Progressive Matrices: Aferition studies]. Unpublished Doctoral Dissertation. 
Universidade de Coimbra. Coimbra, Portugal.

Stainton, T., \& Besser, H. (1998). The positive impact of children with an intellectual disability on the family. Journal of Intellectual and Developmental Disability, 23, 57-70. doi: $|0.1080 /| 366825980003358$ |

Tedeschi, R. G., \& Calhoun, L. G. (2004). Posttraumatic growth: Conceptual foundations and empirical evidence. Psychological Inquiry, I5, I-I8. doi:I0.1207/s I5327965pliI50I_0I

The WHOQOL Group (1994). Development of the WHOQOL: Rationale and current status. International Journal of Mental Health, 23(3), 24-56.

van Gent, R., van Essen, L., Rovers, M., Kimpen, J., van der Ent, C., \& de Meer, G. (2007). Quality of life in children with undiagnosed and diagnosed asthma. European Journal of Pediatrics, 166, 843-848. doi: 10.1007/s0043I-006-0358-y

Varni, J. W., Burwinkle, T. M., \& Lane, M. M. (2005). Health-related quality of life measurement in pediatric clinical practice: An appraisal and precept for future research and application. Health and Quality of Life Outcomes, 3, 34. doi:10.1 186/1477-7525-3-34

Vila, G., Hayder, R., Bertrand, C., Falissard, B., de Blic, J., Mouren-Simeoni, M. C., \& Scheinmann, P. (2003). Psychopathology and quality of life for adolescents with asthma and their parents. Psychosomatics, 44, 319-328. doi:10.1 176/appi.psy.44.4.319

Wallander, J. L., Pitt, L. C., \& Mellins, C. A. (1990). Child functional independence and maternal psychosocial stress as risk factors threatening adaptation in mothers of physically or sensorially handicapped children. Journal of Consulting and Clinical Psychology, 58, 8I8-824. doi: 10.1037/0022-006X.58.6.818

Wallander, J. L., Varni, J. W., Babani, L., Banis, H. T., \& Wilcox, K. T. (1989). Family resources as resistance factors for psychological maladjustment in chronically ill and handicapped children. Journal of Pediatric Psychology, 14, I57-I73. doi:I0.1093/jpepsy/14.2.157

Walsh, F. (2002). A family resilience framework: Innovative practice applications. Family Relations, 5 I, |30-| 37. doi: |0. I | | |/j. I74|-3729.2002.00 |30.x 